\title{
Sudden Visual Deterioration as the First Symptom of Chronic Kidney Failure
}

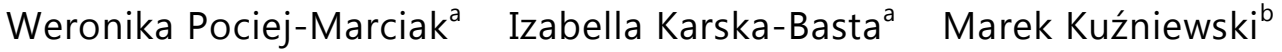 \\ Agnieszka Kubicka-Trząska ${ }^{a}$ Bożena Romanowska-Dixon ${ }^{a}$ \\ Departments of ${ }^{a}$ Ophthalmology and ${ }^{b}$ Nephrology, Jagiellonian University Medical \\ College, Kraków, Poland
}

\section{Key Words}

Hypertensive crisis · Hypertensive choroidopathy · Hypertensive retinopathy · Chronic renal failure

\begin{abstract}
Purpose: We report here a unique case of a sudden loss of vision as the first symptom of an advanced chronic nephropathy. Methods and Results: A 25-year-old man was referred to the Department of Ophthalmology with sudden visual deterioration presumptively diagnosed as bilateral retinitis. The patient had never been under any medical care before and had never had any clinical signs of any chronic disease. He underwent an ophthalmic examination with optical coherence tomography (OCT). Based on the clinical features, OCT scans and systemic blood pressure (BP) assessment $(225 / 145 \mathrm{~mm} \mathrm{Hg}$ ), the patient was definitely diagnosed with hypertensive retinopathy and choroidopathy due to hypertensive crisis. After urgent diagnostic procedures, the patient was diagnosed with a chronic kidney disease at stage 5 in the course of chronic glomerulonephritis. Immediately, a renal replacement therapy was started and the patient was qualified for renal transplantation. Conclusion: Adolescents with an unclear picture of retinal lesions, who have neither a history nor clinical signs of a systemic disease, should undergo careful systemic screening with BP assessment. A sudden deterioration of vision may be the first symptom of a previously undiagnosed severe systemic disease (very rare chronic) that requires immediate treatment.
\end{abstract}


Pociej-Marciak et al.: Sudden Visual Deterioration as the First Symptom of Chronic Kidney Failure

\section{Introduction}

Hypertensive crisis (HC) is a life-threatening condition resulting from an acute elevation in blood pressure (BP) in which the diastolic BP levels are usually over $120 \mathrm{~mm} \mathrm{Hg} \mathrm{[1].} \mathrm{It} \mathrm{is} \mathrm{a}$ common medical problem and accounts for $27.5 \%$ of all medical emergencies at the Emergency Department [2]. HC can manifest as hypertensive urgency without target-organ damage or as hypertensive emergency with target-organ damage (malignant hypertension, $\mathrm{MH}$ ). Undiagnosed systemic hypertension may lead to cardiovascular complications, damage to the capillaries of the central nervous system, kidney glomeruli, retina, choroid and optic nerve $[3,4]$. Impairment of vision occurs in $35-60 \%$ of all patients with $\mathrm{MH}$ [2]. We report here the case of a young patient with sudden visual deterioration due to chronic glomerulonephritis who did not have any other symptoms characteristic of chronic renal disease.

Remarkably, to the best of our knowledge, there have been only few papers published on vision loss as the first symptom of a previously undiagnosed chronic kidney failure.

\section{Methods and Results}

A 25-year-old man, with a presumptive diagnosis of bilateral retinitis was admitted to our Department of Ophthalmology as an emergency. The patient was admitted with a 4-day history of visual deterioration, accompanied by symptoms of upper respiratory tract infection. A similar episode had occurred a month earlier and subsided spontaneously. It should be stressed that the patient had never been under any medical care before.

Ophthalmic examination revealed best corrected visual acuity (BCVA) 0.25 in the right eye (RE) and 0.32 in the left eye (LE). Intraocular pressure was normal in both eyes. Slitlamp examination showed no abnormalities. Indirect ophthalmoscopy revealed bilateral macular oedema, numerous flame-shaped haemorrhages, hard exudates and 'cotton wool spots' located in the posterior pole of the fundus. Disseminated foci of hyperpigmentation arranged linearly on the periphery were present and indistinct optic disc borders at the nasal aspect were observed (fig. 1). OCT scans revealed subfoveal retinal detachment and macular oedema in both eyes (fig. 2). Central retinal thickness was $504 \mu \mathrm{m}$ in the RE and $804 \mu \mathrm{m}$ in the LE. A full-system examination revealed a high BP (225/145 mm Hg). The patient was diagnosed with HC and referred as an emergency to the Internal Medicine Department for further management. Laboratory tests showed elevated serum creatinine level (566.0 $\mu \mathrm{mol} / \mathrm{l}$; norm: 62-106 $\mu \mathrm{mol} / \mathrm{l}$ ), decreased glomerular filtration rate (GFR; $11 \mathrm{ml} / \mathrm{min} / 1.73$ $\mathrm{m}^{2}$; norm: $>60 / \mathrm{min} / 1.73 \mathrm{~m}^{2}$ ), serum urea level $19.4 \mathrm{mmol} / \mathrm{l}$ (norm: 2.76-8.07 mmol/l), serum potassium level $5.5 \mathrm{mmol} / \mathrm{l}$ (norm: 3.5-5.1 mmol/l) and proteinuria $5.28 \mathrm{~g} / \mathrm{l}$. A definitive diagnosis of a chronic kidney disease at stage 5 in the course of chronic glomerulonephritis was established. The patient was given anti-hypertensive medication and was referred to the Nephrology Department, where an urgent renal replacement therapy (haemodialysis) was started. The patient was treated with prednisolone at the starting dose of 20 mg per day.

Due to the enlarged size of the kidneys on ultrasonography (right kidney: $107 \times 45 \mathrm{~mm}$, right renal cortical thickness: $14-16 \mathrm{~mm}$, left kidney: $103 \times 40 \mathrm{~mm}$, left renal cortical thickness: $14-17 \mathrm{~mm}$ ) and the presence of nephrotic-level proteinuria of $5.28 \mathrm{~g} / \mathrm{l}$, as well as active urine sediment, it was decided to carry out a diagnostic kidney biopsy, which confirmed advanced chronic nephropathy (chronic inflammatory glomerular sclerosis lesions).

The follow-up ophthalmic examination performed 3 months after the diagnosis showed an improvement in BCVA to 0.8 in each eye. On fundoscopy, a regression of clinical features 
Pociej-Marciak et al.: Sudden Visual Deterioration as the First Symptom of Chronic Kidney Failure

of hypertensive retinochoroidopathy was observed (fig. 3), and the renal parameters had improved except for creatinine and GFR. Blood tests revealed the following results: serum creatinine level $569.0 \mu \mathrm{mol} / \mathrm{l}$, GFR $11 \mathrm{ml} / \mathrm{min} / 1.73 \mathrm{~m}^{2}$, serum urea level $10.8 \mathrm{mmol} / \mathrm{l}$, serum potassium level $4.95 \mathrm{mmol} / \mathrm{l}$ and proteinuria $4.1 \mathrm{~g} / \mathrm{l}$. The BP on medication (Amlopin - amlodipinum $2 \times 10 \mathrm{mg}$, Nebilenin - nebivololum $1 \times 5 \mathrm{mg}$, Furosemid - furosemidum $2 \times 20$ $\mathrm{mg}$ ) was $150 / 80 \mathrm{~mm}$ Hg. The OCT scans showed the decreased central retinal thickness (281 $\mu \mathrm{m}$ in the RE, and $262 \mu \mathrm{m}$ in the LE) with total regression of cystoid macular oedema (fig. 4).

Because of the patient's young age, after achieving the compensation of metabolic chronic uraemia, the patient qualified for renal transplantation.

\section{Discussion}

Hypertensive choroidopathy typically occurs in adolescents with HC, in acute hypertension in pregnant women with preeclampsia and eclampsia, as well as in patients with pheochromocytoma and kidney diseases [3-7]. The essence of choroidopathy is the absence of choroidal capillary circulation, leading to the ischemic necrosis of the choroid and retinal pigment epithelium (RPE). On fundoscopy, 'Elschnig spots' are present as a result of infarct lesions in the choroid and RPE and 'Siegrist's bands' - linearly arranged spots along the choroidal vessels representing areas of fibrosing necrosis. Local RPE detachment and exudative retinal detachment may also occur in the course of hypertensive choroidopathy [8].

The visual disturbances may be the first clinical sign of MH. Tibbetts et al. [7] described the case of a 7-year-old child, in whom deterioration of vision was observed during a school screening exercise, and then he was diagnosed with systemic hypertension due to pheochromocytoma. Malhotra et al. [5] presented the case of an 18-year-old female patient with hypertension associated with bilateral renal artery stenosis who, as the first symptom, had complained of bilateral deterioration of vision. Similarly, Wijaya and Siregar [9] drew attention to the fact that the occurrence of $\mathrm{HC}$ in adolescents may be associated with renovascular hypertension. Kovach [10] described the case of an 18-year-old female patient, who was undergoing treatment for kidney damage due to focal segmental glomerulonephritis and underwent ophthalmic examination because of a sudden bilateral visual loss. Kovach underlines that the pathogenesis of glomerulonephritis may be autoimmune in nature; however, in the image of the ocular fundus of the examined patient, there were no signs of inflammation [10]. Hypertensive choroidopathy is most frequently found in adolescents; however, noteworthy is a study by Hirano et al. [4], in which the authors presented the case of a 50-yearold male patient with a long-standing history of hypertension who demonstrated a sudden vision loss with fundus signs consistent with that of hypertensive choroidopathy.

The treatment of hypertensive choroidopathy is based on lowering the BP through the administration of antihypertensive drugs [10]. There have also been attempts of sub-Tenon injection of corticosteroids [11]. Ocular signs such as papilloedema or retinal detachment most often subside a few weeks after normalization of the systemic BP. However, to preserve vision in these cases, a prompt diagnosis of $\mathrm{MH}$ is essential [4].

In the presented case, the visual deterioration was the first clinical symptom of a previously undiagnosed chronic kidney disease.

In summary, based on the literature review and on our observations, it can be concluded that patients complaining of visual disturbances with non-characteristic retinal lesions should be screened for systemic hypertension. In the event of a HC, BP should be aggressively but gradually normalized to the levels appropriate to the patient's age. At the same time, a 
Pociej-Marciak et al.: Sudden Visual Deterioration as the First Symptom of Chronic Kidney Failure

quick analysis of the cause behind a HC should be performed, which should include fundoscopy, in order to find its cause and to start treatment as soon as possible.

\section{Statement of Ethics}

The authors have no ethical conflicts to disclose.

\section{Disclosure Statement}

The authors declare that they have no financial interests and nothing to disclose regarding this case report.

\section{References}

$\rightarrow 1$ Vilela-Martin JF, Vaz-de-Melo RO, Kuniyoshi CH, et al: Hypertensive crisis: clinical-epidemiological profile. Hypertens Res 2011;34:367-371.

$\longrightarrow 2$ Kitiyakara C, Guzman NJ: Malignant hypertension and hypertensive emergencies. J Am Soc Nephrol 1998;9:135.

-3 Bourke K, Pate MR, Prisant LM, Marcus DM: Hypertensive choroidopathy. J Clin Hypertens (Greenwich) 2004;6:471-472.

-4 Hirano Y, Yasukawa T, Ogura Y: Bilateral serous retinal detachments associated with accelerated hypertensive choroidopathy. Int J Hypertens 2010;1:1-4.

5 Malhotra SK, Gupta R, Sood S, et al: Bilateral renal artery stenosis presenting as hypertensive retinopathy and choroidopathy. Indian J Ophthalmol 2002;50:221-223.

-6 Nakamura T, Miyauchi O, Mizota A, Adachi-Usami E: Bilateral leopard spot fundus of hypertensive choroidopathy and its visual function. Ophthalmologica 2002;216:228-229.

7 Tibbetts MD, Wise R, Forbes B, et al: Hypertensive retinopathy in a child caused by pheochromocytoma: Identification after a failed school vision screening. J AAPOS 2012;16:97-99.

8 Parke DW: Basic and Clinical Science Course. Section 12: Retina and Vitreous. San Francisco, American Academy of Ophthalmology, 2005-2006.

-9 Wijaya I, Siregar P: Hypertensive crises in the adolescent: evaluation of suspected renovascular hypertension. Acta Med Indones 2013;45:49-54.

10 Kovach JL: Hypertensive optic neuropathy and choroidopathy in an 18-year-old woman with renal failure. Retin Cases Brief Rep 2010;4:187-189.

11 Mansour AM, Medawar WA: Periocular corticosteroid in malignant hypertensive and preeclampsic choroidopathy. Retin Cases Brief Rep 2009;3:144-146. 


\section{Case Reports in \\ Ophthalmology}

\begin{tabular}{l|l}
\hline Case Rep Ophthalmol 2015;6:394-400 \\
\hline DOI: 10.1159/000442182 & $\begin{array}{l}\text { C } 2015 \text { The Author(s). Published by S. Karger AG, Basel } \\
\text { www.karger.com/cop }\end{array}$ \\
\hline
\end{tabular}

Pociej-Marciak et al.: Sudden Visual Deterioration as the First Symptom of Chronic Kidney Failure

a

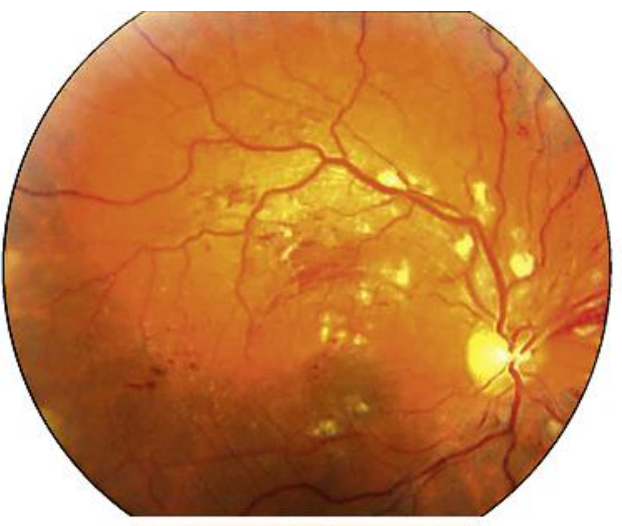

b

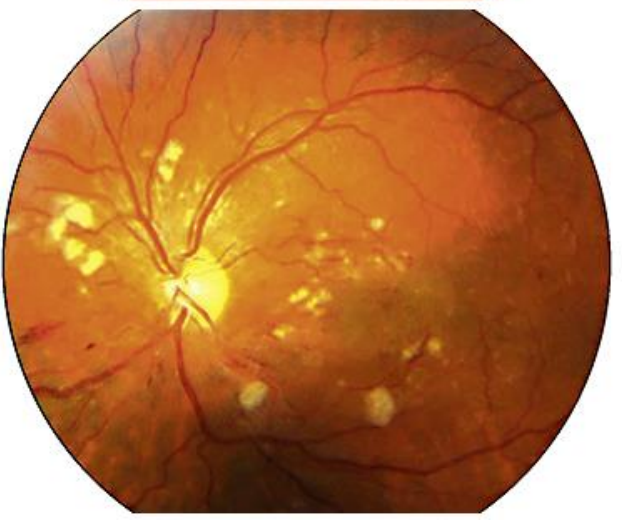

Fig. 1. a, b Colour pictures of both eyes: 'flame-shaped' haemorrhages and hard exudates in the posterior pole, 'cotton wool spots' located around the optic disc and disseminated foci of hyperpigmentation arranged linearly on the periphery, indistinct optic disc borders.

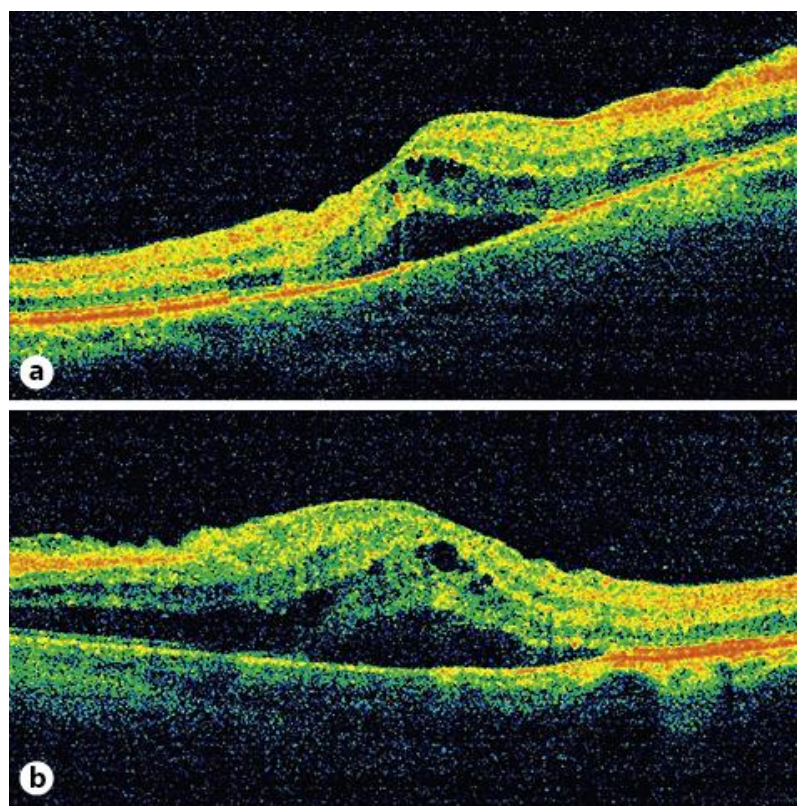

Fig. 2. a, b OCT scans of the macula in both eyes: subfoveal fluid and macular oedema are present. 
Case Reports in

Ophthalmology
Case Rep Ophthalmol 2015;6:394-400

\begin{tabular}{l|l}
\hline DOI: $10.1159 / 000442182$ & (C) 2015 The Author(s). Published by S. Karger AG, Basel
\end{tabular} www.karger.com/cop

Pociej-Marciak et al.: Sudden Visual Deterioration as the First Symptom of Chronic Kidney Failure

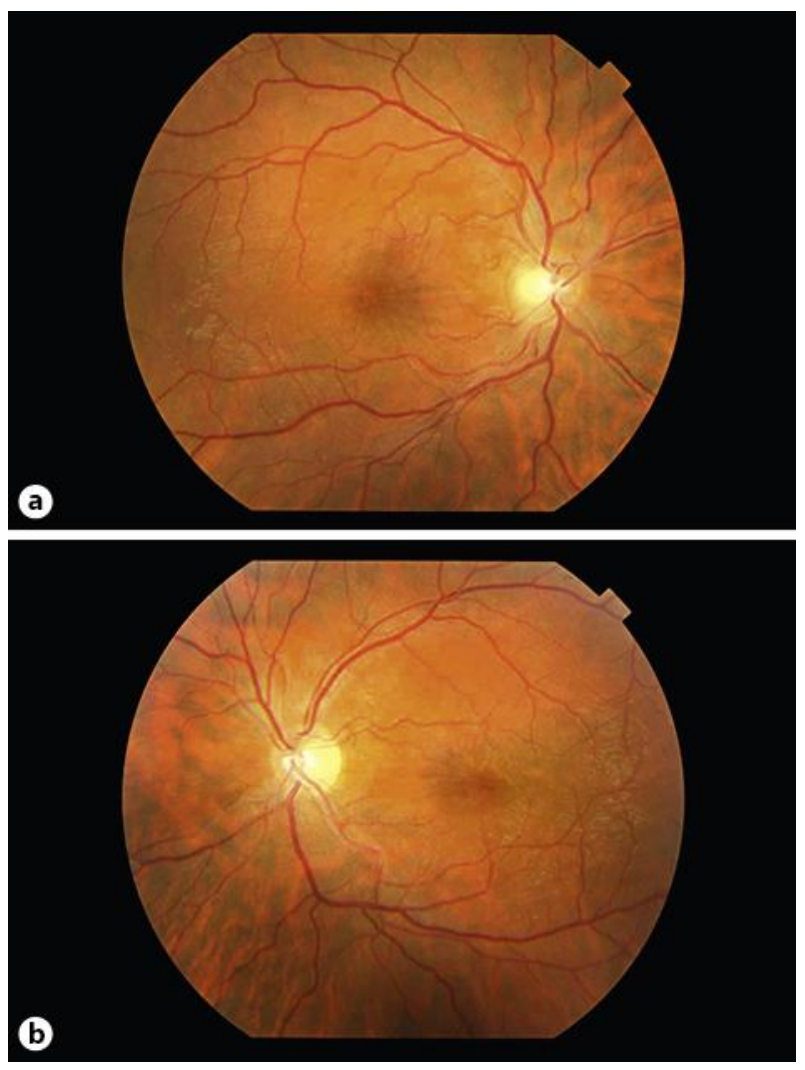

Fig. 3. a, b Colour pictures of both eyes 3 months after treatment was started: no signs of hypertensive choroidopathy and retinopathy are observed. Epiretinal membranes are present. 
Case Reports in

Ophthalmology
Case Rep Ophthalmol 2015;6:394-400

\begin{tabular}{l|l}
\hline DOI: $10.1159 / 000442182$ & C 2015 The Author(s). Published by S. Karger AG, Basel
\end{tabular} www.karger.com/cop

Pociej-Marciak et al.: Sudden Visual Deterioration as the First Symptom of Chronic Kidney Failure

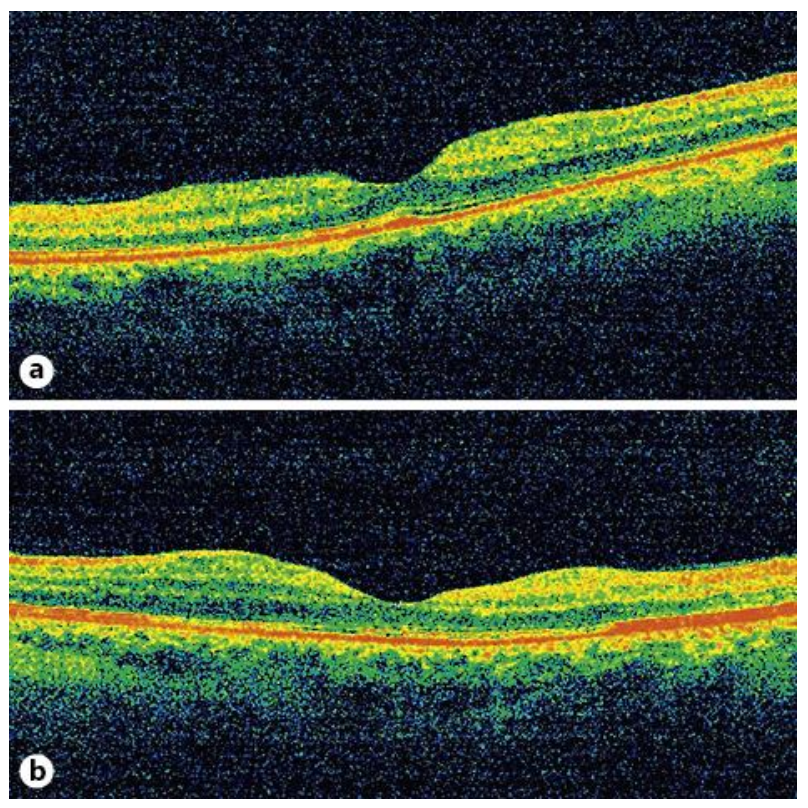

Fig. 4. a, b OCT scans of macula show complete regression of subretinal and intraretinal fluid with bilateral epiretinal membranes. 